Check for updates

Cite this: RSC Adv., 2019, 9, 2525

Received 14th November 2018

Accepted 4th January 2019

DOI: $10.1039 / c 8 r a 09318 k$

rsc.li/rsc-advances

\section{Sequential ligand- and structure-based virtual screening approach for the identification of potential G protein-coupled estrogen receptor-1 (GPER-1) modulators $\dagger$}

\author{
Shafi Ullah Khan, (DD ${ }^{a}$ Nafees Ahemad, ${ }^{\text {ab }}$ Lay-Hong Chuah, ${ }^{\text {ac }}$ Rakesh Naidu ${ }^{d}$ \\ and Thet Thet Htar (D) *a
}

G protein-coupled estrogen receptor-1 (GPER-1) is a seven transmembrane receptor, responsible for mediating rapid estrogen signaling in many physiological responses in reproductive, nervous, endocrine, immune and cardiovascular systems. Due to unavailability of the crystal structure of GPER-1, we have performed sequential ligand-based virtual screening (LBVS) and structure-based screening (SBVS) to identify potential GPER-1 modulators. LBVS and SBVS approaches were validated retrospectively using the Receiver Operating Curve (ROC) plot and the early Enrichment Factor (EF). LBVS was performed based on a GPER-1 agonist, G1, as a query model for screening of the eMolecules library using the Rapid Overlay of Chemical Structure (ROCS) and the electrostatic potential screening (EON) approaches. Topscored hits from LBVS were further screened by SBVS. SBVS was based on generating homology models of GPER-1 and subsequent molecular docking studies. Using Chemguass4 score, we filtered the final hits with the higher score in comparison to G1 (Chemguass4 score $=-11.575$ ). The top-ranked hits were clustered based on similarity in their scaffolds. Prospective validation was performed by evaluating the antiproliferative activity of synthesized compounds (SKO and SKOP) which were representative of top hits obtained from our virtual screening approach.

\section{Introduction}

G protein-coupled receptors (GPCRs) are the most significant cell surface receptor superfamily, composed of approximately 900 members. The structural diversity of GPCRs and their physiological functions make them primary targets for therapeutic drugs. GPCRs play a crucial role in many physiological functions as well as in multiple diseases. ${ }^{1}$ Although at least 500 GPCRs have been identified as therapeutically relevant targets, to-date only a few GPCRs are characterized in apo-form or complex with ligands. ${ }^{2}$

A member of the GPCR family, named G protein-coupled estrogen receptors (GPER-1/GPR30) has been recognized as

${ }^{a}$ School of Pharmacy, Monash University Malaysia, Jalan Lagoon Selatan, Bandar Sunway, 47500 Subang Jaya, Selangor, Malaysia.E-mail: thet.thet.htar@monash.edu ${ }^{b}$ Tropical Medicine and Biology Multidisciplinary Platform, Monash University Malaysia, Jalan Lagoon Selatan, Bandar Sunway, 47500 Subang Jaya, Selangor, Malaysia

'Advanced Engineering Platform, Monash University Malaysia, Jalan Lagoon Selatan, Bandar Sunway, 47500 Subang Jaya, Selangor, Malaysia

${ }^{d} J$ effrey Cheah School of Medicine and Health Sciences, Monash University Malaysia, Jalan Lagoon Selatan, Bandar Sunway, 47500 Subang Jaya, Selangor, Malaysia

$\dagger$ Electronic supplementary information (ESI) available. See DOI: $10.1039 / \mathrm{c} 8 \mathrm{ra} 09318 \mathrm{k}$ a novel membrane estrogen receptor. It mediates rapid estrogen signaling in many physiological responses in reproductive, nervous, endocrine, immune and cardiovascular systems. Furthermore, its contribution to the progression of several types of tumors has been reported. ${ }^{3}$ Due to its involvement in transactivation of the epidermal growth factor receptor (EGFR), the activation of the mitogen-activated protein kinase (MAPK), phosphoinositide3-kinase (PI3K) transduction pathways, the stimulation of adenyl cyclase and the mobilization of intracellular calcium, ${ }^{4}$ GPER-1 has attracted attention as a promising new drug target.

Computer-aided virtual screening is one of the valid approaches used in the drug discovery process. The two main strategies commonly applied in computer-aided drug discovery are structure-based virtual screening (SBVS) and ligand-based virtual screening (LBVS). SBVS depends on the structure of the target and its interactions with the ligands while LBVS depends on the structural information and molecular properties of known ligands. ${ }^{5,6}$ Both LBVS and SBVS have been proven to be cost-effective and efficient in generating the leads for further medicinal chemistry development, without requiring complex infrastructures. These methods have been used in search of new drug candidates for therapeutic targets. ${ }^{?}$ 
In the absence of a crystal structure of the target protein, computer-aided methodologies have become increasingly useful in identifying the atomic structure of membrane protein from the homologous structure. ${ }^{8}$ Virtual screening aims to reduce the enormous virtual space of chemical compounds to a manageable number of compounds for further screening against biological targets. In the past few years, in silico virtual screening techniques have been successfully applied in the discovery of new classes of inhibitors for several key therapeutic targets..$^{\mathbf{9} 10}$ Interestingly, the classes of compounds discovered usually differ considerably from the drugs already available.

In this study, we used a sequential application of ligand- and structure-based virtual screening for the identification of GPER1 modulators. The LBVS process can be based on several techniques, including molecular similarity methods, pharmacophore models or machine learning methods. ${ }^{11}$ These approaches do not take the target structure directly into account but instead based on the assumption that compounds with a similar topology will present similar biological activity. They are often applied in combination with structure-based approaches to identify potential bioactive hits that can then be fed into docking experiments. ${ }^{12,13}$ Although various virtual screening procedures have been reported in the literature for different target proteins, ${ }^{\mathbf{1 4 - 1 6}}$ this is the first study of ligandbased virtual screening followed by the structure-based virtual screening of large database compounds for GPER-1 modulating activity. Moreover, we validated our virtual screening model retrospectively and prospectively in order to demonstrate the robustness of the model.

\section{Materials and methods}

\subsection{Retrospective validation of ligand- and structure-based virtual screening approach}

2.1.1 Preparation of active and decoy GPER-1 datasets. Fifteen different active GPER-1 ligands were retrieved from previously published literature. DecoyFinder v 2.0 was used to find out chemically distinct decoy GPER-1 for all selected active GPER-1. To avoid biases, and to mimic real decoy GPER-1, a drug-like subset of ZINC15 was used as a search database. ${ }^{17,18}$ Stringent criteria were followed for the generation of decoys GPER-1 ligands based on five physical descriptors as per directory of the useful decoys (DUD) database. ${ }^{19}$ In the following criteria, the threshold for both active ligand versus decoy and decoy versus decoy, Tanimoto were set as 0.90 while acceptors and donors of hydrogen bonds were set as \pm 0 . Other physical parameters such as molecular weight and rotational bond were set as $\pm 40 \mathrm{Da}$ and \pm 0 respectively. ${ }^{17}$ After generation of prerequisite GPER-1 datasets, retrospective validation of both LBVS and SBVS were carried out using the Receiver Operating Characteristic (ROC) plot and the early enrichment factor (EF) at $0.5 \%, 1 \%$, and $2 \%$ as described in previous reports. ${ }^{20,21}$ Enrichment in true positives (TP) is reported at the false positive rate $(\mathrm{FPR})$ of $1 \%\left(\mathrm{EF}_{1 \%}\right)$ as follows: $\mathrm{EF} \%=\mathrm{TP} / \mathrm{FPR} \%$. Early enrichment at $0.5 \%, 1 \%$, and $2 \%$ rate were computed for each virtual screening parameter using Rocker tool. ${ }^{22}$

\subsection{Ligand-based virtual screening (LBVS)}

We performed LBVS by using two approaches; shape-based virtual screening and electrostatic potential similarity calculation.

2.2.1 Shape-based virtual screening. Shape-based virtual screening was carried out using a rapid overlay of 3D chemical structures (ROCS v3.2.2) tool of OpenEye Scientific Software. ${ }^{23}$ For ROCS screening, GPER-1 selective agonist, G-1 (1$((3 a R, 4 S, 9 b S)$-4-(6-bromobenzo[ $d][1,3]$ dioxol-5-yl)-3a,4,5,9b-tetrahydro-3H-cyclopenta[c]quinolin-8-yl)ethan-1-one) was selected for the generation of ROCS model. The multiple conformations of G-1 were calculated using OMEGA in default settings of maximum 200 conformers per molecule ${ }^{24}$ and the lowest energy conformer of G-1 was used for the generation of final ROCS overlay model.

Generated ROCS models were employed to screen chemical databases 'eMolecules v2017.1' (containing about 7.2 million compounds, eMolecules Inc., La Jolla, CA, USA). ${ }^{25}$ In ROCS, TanimotoCombo which quantifies both ShapeTanimoto (molecular shape overlay) and ColorTanimoto (chemical functionality overlaps), was selected as a scoring parameter. Compounds having the TanimotoCombo score higher than 1.2 were subjected to further screening.

2.2.2 Electrostatic potential similarity. Aligned compounds obtained from ROCS were further analyzed for electrostatic potential similarity with the EON v 2.2.0. ${ }^{26}$ ET_Comb score, which is a combination of ShapeTanimoto (ST) and PB Electrostatic Tanimoto (ET_pb) score, was used to narrow down the hit compounds. Compounds with the ET_Comb score higher than 1 were selected for further screening.

\subsection{Structure-based virtual screening (SBVS)}

SBVS requires pre-requisite target protein structure, used in the process of virtual screening. Structure-based virtual screening was performed using homology modeling and molecular docking studies.

2.3.1 Homology modeling. To date, the 3D structure of the GPER-1 is not available. Homology modeling technique was used to generate the 3D structure of the GPER-1. Previously, many studies have reported homology models of GPER-1 using various templates. We generated a homology model of GPER-1 using both single template (based on previously reported templates) and multi-template approaches. Uniport ID Q99527 was used to fetch a full-length amino acid sequence of GPER-1. A single template homology modeling was carried out using Modeller v9.15 built-in tool in Discovery Studio as shown in Fig. 1. The best model with the lowest Discrete Optimized Potential Energy (DOPE) score was selected for further studies. ${ }^{27}$

Besides the Modeller tool, several online servers, SWISSMODEL, I-TASSER, and GPCR-MODSIM were used for the generation of GPER-1 models. ${ }^{28-30}$ GPER-1 models were generated using default settings available both in SWISS-MODEL and I-TASSER servers. In the case of GPCR-MODSIM server, settings were customized to build homology model based on highest sequence similarity for each topological region (N-t, TM1 IL1, TM2, EL1, TM3, IL2, TM4, EL2, TM5, IL3, TM6, EL3, TM7, C-t). 


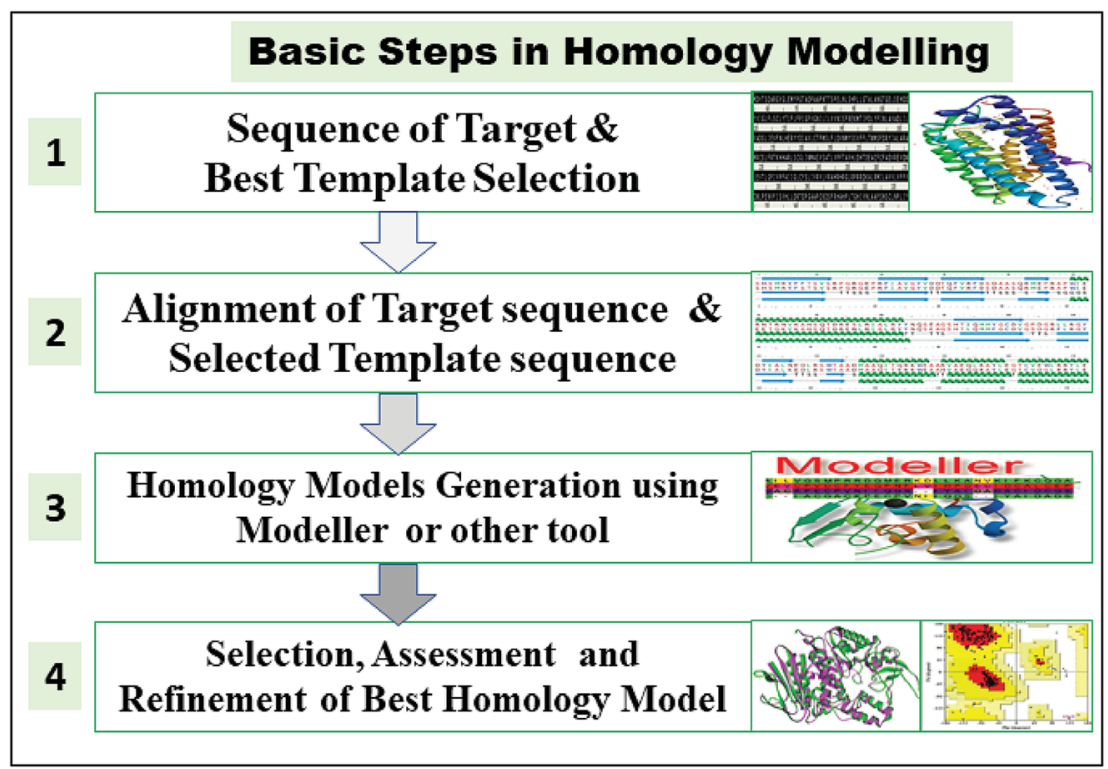

Fig. 1 Stepwise workflow for generating homology model of GPER-1 in Discovery Studio.

After generation of GPER-1 homology model, RAMPAGE and ModRefiner server were used for assessment and refinement of GPER-1 homology models. ${ }^{31}$

2.3.2 Binding site identification of protein. Computed Atlas of Surface Topography of proteins (CASTp) server was employed for predicting the catalytic sites in GPER-1 receptor. ${ }^{32}$

2.3.3 Docking-based virtual screening. Fig. 2 shows the workflow of docking-based virtual screening. Briefly, molecular docking calculation of all virtual hits from ROCS and EONS screening was carried out using FRED v3.2.0 built-in utility of OpenEye Scientific Software. ${ }^{3,34}$ Prior to docking, GPER-1 structure was prepared using a pdb2receptor tool in OEDocking and optimized at $\mathrm{pH}$ 7.0. Multi-conformers of hits were generated by OMEGA2.5.1. ${ }^{24}$ Default settings of OMEGA were kept in the generation of conformers (maximum 200 conformers per molecule). The binding site for docking calculation was defined within $10 \AA$ of the active site region. Binding affinity was predicted by FRED default parameters. Previously reported GPER-1 ligands (see ESI $\dagger$ for structures details) were docked before the docking of database compounds. After protocol optimization, ROCS and EON hits were docked using the same protocol as mentioned earlier. Maximum of 10 poses for each compound were generated, and best-hits were selected based on lowest Chemgauss4 score. Binding orientation of docked poses was visualized using Discovery Studio Visualizer and Chimera v1.1. ${ }^{35}$

\subsection{Prospective validation of ligand- and structure-based virtual screening approach}

After clustering of hit compounds, two compounds containing the top hit scaffold were synthesized and tested for antiproliferative activities to validate the in silico virtual screening approach prospectively.

2.4.1 Synthesis of compounds containing hit scaffold
2.4.1.1 1-((3aR,4R,9bR)-4-(6-Bromobenzo[d][1,3]dioxol-5yl)-2,3,3a,4,5,9b-hexahydrofuro[3,2-c]quinolin-8-yl)ethan-1-one (SKO). To a solution of 6-bromopiperonal ( $0.343 \mathrm{~g}, 1.5 \mathrm{mmol})$ in acetonitrile $(4 \mathrm{~mL}), p$-aminoacetophenone $(0.202 \mathrm{~g}, 1.5$ $\mathrm{mmol}$ ) was added and stirred at room temperature. Then 2,3dihydrofuran $(0.210 \mathrm{~g}, 3 \mathrm{mmol})$ and scandium(iii) trifluoromethanesulfonate $\left(\mathrm{Sc}(\mathrm{OTf})_{3}\right)(0.074 \mathrm{~g}, 0.15 \mathrm{mmol})$ were added to the mixture. The reaction mixture was then irradiated at $100{ }^{\circ} \mathrm{C}$ for 20 minutes using microwave system (150 $\mathrm{W})$. Upon completion of reaction, the volatiles were removed in vacuo and water was added to the mixture. The mixture was then extracted with ethyl acetate. The organic layers were pooled and dried over anhydrous magnesium sulphate $\left(\mathrm{MgSO}_{4}\right)$. Purification by silica gel flash column chromatography using hexane-ethylacetate $(8: 2 \mathrm{v} / \mathrm{v})$ gave the title compound $(0.460 \mathrm{~g}, 74 \%$ yield $)$. Further recrystallization from $\mathrm{CH}_{3} \mathrm{CN}-\mathrm{H}_{2} \mathrm{O}(2: 1 \mathrm{v} / \mathrm{v})$ gave diastereomerically pure compound (SKo) as a white solid product, mp: $204-206{ }^{\circ} \mathrm{C}$.

${ }^{1} \mathrm{H}$ NMR (DMSO- $\left.d_{6}, 300 \mathrm{MHz}\right): \delta 7.81(\mathrm{~s}, 1 \mathrm{H}, \mathrm{N}-\mathrm{H}), 7.65$ (dd, $J$ $=2.0 \mathrm{~Hz}, 8.6 \mathrm{~Hz}, 1 \mathrm{H}, \mathrm{H}-7), 7.27(\mathrm{~s}, 1 \mathrm{H}, \mathrm{H}-9), 7.18(\mathrm{~s}, 1 \mathrm{H}, \mathrm{H}-19)$, $6.78(\mathrm{~s}, 1 \mathrm{H}, \mathrm{H}-15), 6.73(\mathrm{~d}, J=8.4 \mathrm{~Hz}, 1 \mathrm{H}, \mathrm{H}-6), 6.11(\mathrm{~d}, J=$ $8.4 \mathrm{~Hz}, 2 \mathrm{H}, \mathrm{H}-17), 5.16$ (d, $J=7.5 \mathrm{~Hz}, 1 \mathrm{H}, \mathrm{H}-5), 4.94$ (d, $J=3 \mathrm{~Hz}$, 1H, H-3a), 3.66-3.62 (m, 2H, H-12), 2.78 (d, J= $7.5 \mathrm{~Hz}, 1 \mathrm{H}, \mathrm{H}-4)$, 2.50 (s, 3H, H-11), 1.96-1.83 (m, 1H, H-13), 1.45-1.35 (m, 1H, H13). ${ }^{13} \mathrm{C}$ NMR (DMSO- $\left.d_{6}, 75 \mathrm{MHz}\right): \delta 195.79(\mathrm{C}=\mathrm{O}, \mathrm{C}-10), 150.00$ (C-N, C-2), 147.94 (C-O, C-16), 147.67 (C-O, C-18), 133.48 (C-14), 131.19 (C-9), 129.20 (C-8), 127.13 (C-7), 120.96 (C-3), 114.64 (C19), 112.91 (C-20), 112.66 (C-15), 108.80 (C-6), 102.55 (C-17), 74.57 (C-3a), 66.30 (C-12), 54.86 (C-5), 41.34 (C-4), 26.43 (C-11), 24.90 (C-13). FTIR-ATR ( $\left.\mathrm{cm}^{-1}\right): \nu 3343.46$ (NH amine), 3299.77 (N-H str), 2875 (CH-str), 1643.80 ( $\mathrm{C}=\mathrm{O}$, conjugated), 1579.93, 1472.08, 1360.26, 1322.75, 1279.57, 1235.14, 1032.84. HRMS (EIMS) $m / z$ : calcd for $\mathrm{C}_{20} \mathrm{H}_{18} \mathrm{BrNO}_{4}\left[\mathrm{M}+\mathrm{H}^{+}\right]$: 415.0419; found: $415.0427\left[\mathrm{M}+\mathrm{H}^{+}\right]$. 
Generation of Target Protein GPER1

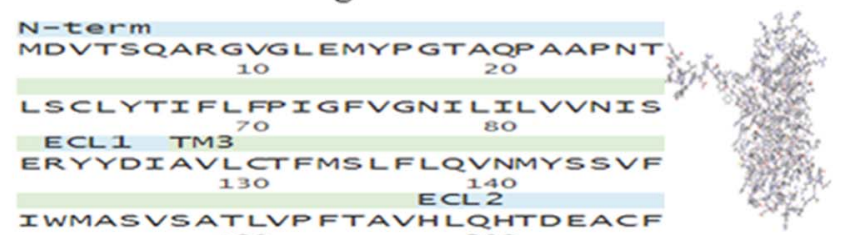

Preparation, Optimization, Assigning Charge and energy minimization of GPER1 using QUACPAC tools

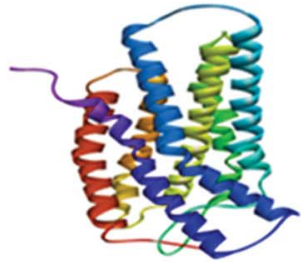

Selection of Active pocket using CASTp / OpenEye tool

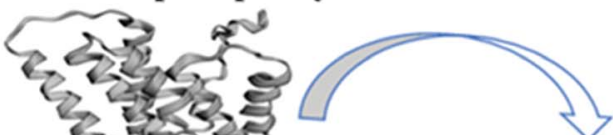

Molecular Docking Calculation using FRED

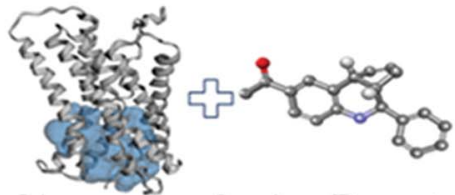

Optimization of Docking protocol using Reported GPER1 ligands

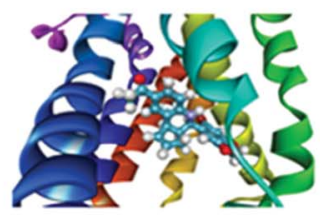

Molecular Docking of Insilico hits obtained from EON and ROCS screening

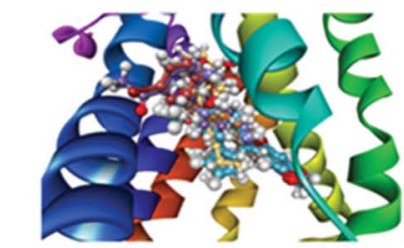

Selection of Best Hits based on ChemGauss4 Score
Selection of ligands

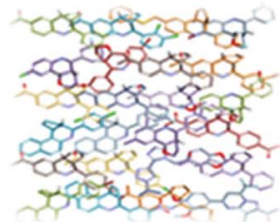

Generation of multi-Conformers of each ligands using OMEGA

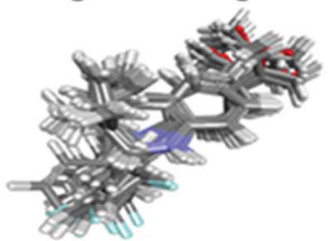

Optimization and Preparation of each Ligands using QUACPAC tool

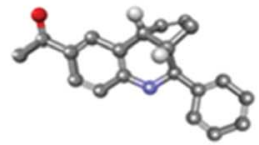

Fig. 2 Step by step workflow for performing docking-based virtual screening.

2.4.1.2 1-((4aR,5R,10bR)-5-(6-Bromobenzo[d][1,3]dioxol-5-yl)3,4,4a,5,6,10b-hexahydro-2H-pyrano[3,2-c]quinolin-9-yl)ethan-1one (SKOP). To a solution of 6-bromopiperonal $(0.343 \mathrm{~g}, 1.5$ $\mathrm{mmol})$ in acetonitrile $(4 \mathrm{~mL}), p$-aminoacetophenone $(0.200 \mathrm{~g}$, $1.5 \mathrm{mmol}$ ) was added and stirred at room temperature. Then 3,4-dihydro- $2 H$-pyran $(0.252 \mathrm{~g}, 3 \mathrm{mmol})$ and scandium(iii) trifluoromethanesulfonate $\left(\mathrm{Sc}(\mathrm{OTf})_{3}\right)(0.074 \mathrm{~g}, 0.15 \mathrm{mmol})$ were added to the mixture. The reaction mixture was then irradiated at $100{ }^{\circ} \mathrm{C}$ for 20 minutes using microwave system $(150 \mathrm{~W})$. Upon completion of reaction, the volatiles were removed in vacuo and water was added to the mixture. The mixture was then extracted with ethyl acetate. The organic layers were combined and dried over anhydrous magnesium sulphate $\left(\mathrm{MgSO}_{4}\right)$. Purification by silica gel flash column chromatography using hexane-ethyl acetate $(8: 2 \mathrm{v} / \mathrm{v})$ gave the title compound $(0.427 \mathrm{~g}, 66 \%$ yield). Further recrystallization from $\mathrm{CH}_{3} \mathrm{CN}-\mathrm{H}_{2} \mathrm{O}(2: 1 \mathrm{v} / \mathrm{v})$ gave diastereomerically pure compound (SKOP) as a yellowish-white solid product, mp: $124-126^{\circ} \mathrm{C}$.

${ }^{1} \mathrm{H}$ NMR (DMSO- $d_{6}, 300 \mathrm{MHz}$ ): $\delta 7.81(\mathrm{~s}, 1 \mathrm{H}, \mathrm{N}-\mathrm{H}), 7.65$ (dd, $1 \mathrm{H}, J=2.0 \mathrm{~Hz}, 8.6 \mathrm{~Hz}, \mathrm{H}-7), 7.27$ (s, 1H, H-9), 7.06 (s, 1H, H-20), 
6.84 (s, 1H, H16), 6.72 (d, 1H, H-6), 6.09 (d, J=13.8 Hz, 2H, H18), 5.18 (d, $J=5.4 \mathrm{~Hz}, 1 \mathrm{H}, \mathrm{H}-5), 4.88$ (d, $J=3 \mathrm{~Hz}, 1 \mathrm{H}, \mathrm{H}-3 \mathrm{a})$, 3.56-3.53 (m, 1H, H-12), 2.50 (s, 3H, H-11), 2.21-2.18 (m, 1H, $\mathrm{H}-4)$, 1.43-1.42 (m, 1H, H-13), 1.10-1.09 (m, 1H, H-14). ${ }^{13} \mathrm{C}$ NMR (DMSO- $\left.d_{6}, 75 \mathrm{MHz}\right): \delta 195.79(\mathrm{C}=\mathrm{O}, \mathrm{C}-10), 150.15(\mathrm{C}-\mathrm{N}, \mathrm{C}-2)$, 147.38 (C-O, C-17), 146.73 (C-O, C-19), 131.39 (C-15), 128.86 (C-9), 127.80 (C-8), 126.06 (C-7), 117.48 (C-3), 113.55 (C-Br, C21), 112.16 (C-16), 108.81 (C-6), 102.00 (C-18), 70.79 (C-3a), 59.60 (C-12), 56.88 (C-5), 34.02 (C-4), 25.87 (C-11), 24.86 (C-13), 18.27 (C-14). FTIR-ATR $\left(\mathrm{cm}^{-1}\right): \nu 3323.81$ (NH amine), 3299.77 (N-H str), $1644.71(\mathrm{C}=\mathrm{O}$, conjugated), 1598.32, 1570.70, 1503.98, 1473.46, 1356.91, 1290.78, 1231.95, 1089.35, 1036.85. HRMS (EI-MS) $m / z$ : calcd for $\mathrm{C}_{21} \mathrm{H}_{20} \mathrm{BrNO}_{4}\left[\mathrm{M}+\mathrm{H}^{+}\right]$: 429.0576, found: $429.0473\left[\mathrm{M}+\mathrm{H}^{+}\right]$.

2.4.2 Antiproliferative activities of hit compounds using GPER-1 specific cell line. Two different human breast cancer cell lines, SK-BR-3 (GPER-1 +ve, ER -ve) and MCF-7 (ER +ve and GPER-1 +ve) were used to investigate the antiproliferative activities of synthesized compounds. ${ }^{37,38}$ SK-BR-3 cells were cultured in McCoy's 5 A (modified) medium supplemented with $10 \%$ fetal bovine serum, $100 \mathrm{U} \mathrm{mL}^{-1}$ penicillin, and $10 \mu \mathrm{g} \mathrm{mL}^{-1}$ streptomycin at $37{ }^{\circ} \mathrm{C}$ in a humidified $5 \%$ carbon dioxide $\left(\mathrm{CO}_{2}\right)$ atmosphere while MCF-7 cells were cultured in Dulbecco's Modified Eagle Medium (DMEM). The optimal number of $7.5 \times$ $10^{3}$ cells per well were seeded in 96-well flat-bottomed plate and incubated for 24 hours. After 24 hours $10 \mu \mathrm{L}$ of each compound at different concentration $(100 \mu \mathrm{M}$ to $0.195 \mu \mathrm{M})$ was added and incubated for 72 hours.

A blank medium was used as a control in the corresponding control wells of the same plate. After incubation for 72 hours, 20 $\mu \mathrm{L}$ of freshly prepared MTT solution was added in the dark into each well and incubated for additional 4 hours at $37{ }^{\circ} \mathrm{C}$ until intracellular purple formazan crystals were visible under a microscope. After that spent media, as well as MTT reagents, was removed and $100 \mu \mathrm{L}$ DMSO as the solubilizing solution was added to dissolve the formazan crystals. The absorbance was measured at $570 \mathrm{~nm}$ and $650 \mathrm{~nm}$ as a reference wavelength using i-Tecan Infinite M200 microplate reader. All experiments were carried out in triplicate. A non-linear regression analysis of the program PRISM v 7.0 (GraphPad, San Diego, California, USA) was used to fit the dose-response curves and to calculate $\mathrm{EC}_{50}$ values.

2.4.3 Molecular docking studies of synthesized compounds. Molecular docking of synthesized compounds was carried out to study the binding affinity with GPER-1. Previously optimized protocol for retrospective validation and virtual screening process was followed (see Section 2.3.3 for details).

\section{Results and discussion}

\subsection{Retrospective validation of ligand- and structure-based virtual screening approach}

In this study, we developed a sequential ligand- and structurebased virtual screening of large database in an attempt to find new GPER-1 modulators. Our virtual screening protocol was validated by retrospective statistical metrics and prospective biological assay. To date, no GPER-1 specific Decoy dataset is available in the directory of useful decoys, enhanced (DUD-E) (http://dude.docking.org/) which contains actives and decoys for 102 different targets. ${ }^{19}$ Therefore, we generated a total of 509 decoys based on the fifteen active GPER-1 ligands using DecoyFinder V 2.0. These decoys have the same physical parameters as actives, but they are chemically distinct in order to act as false positives. Full detail of decoys is shown in the ESI. $\dagger$

The three-dimensional structure of G1 was then generated to use as a query model. To mimics the co-crystallographic ligand, various conformers of G1 were generated using OMEGA 2.5. The best conformer which can discriminate the active GPER-1 ligands from decoy GPER-1 was selected based on the ShapeTanimoto, ColorTanimoto and TanimotoCombo parameters (Fig. 3A and S1 $\dagger$ ). The area under the curve (AUC) and the early
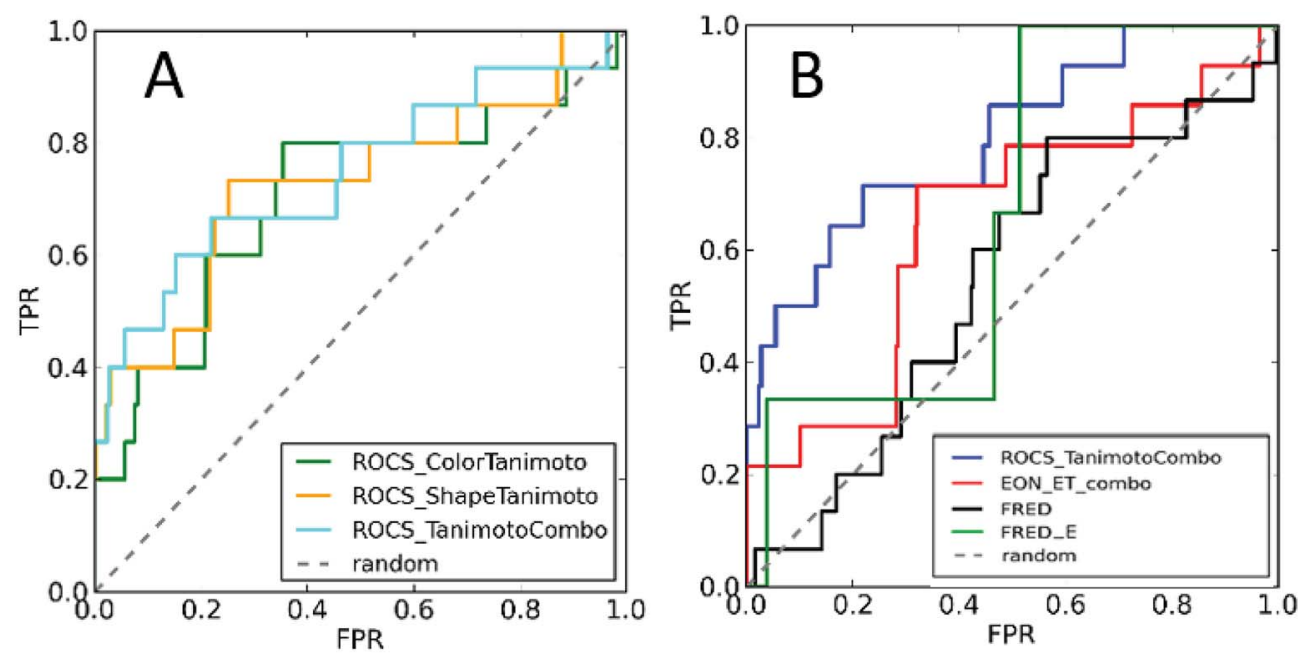

Fig. 3 ROC plots. (A) The ROC-AUC virtual screening-based on TanimotoCombo, ColorTanimoto, and ShapeTanimoto of G-1. (B) Comparison of the ROC-AUC plots: TanimatoCombo score (blue), electrostatic potential (red), SBVS approach using the whole GPER-1 dataset (black) and SBVS approach using only selective GPER-1 ligands (green). 
enrichment factor $(\mathrm{EF})$ at $0.5 \%, 1 \%$, and $2 \%$ rate were used to evaluate the overall performance of virtual screening of query model.

Fig. 3A showed the ROC plots of conformer 1 representing the fraction of active GPER-1 ligand (true positives, TP) on the $y$ axis versus the fraction of decoys (false positives rate, FPR) on the $x$-axis for each LBVS parameter. Among all, the G1 conformer 1 presented the overall good performance having the highest AUC of 0.797 which indicated that $79.7 \%$ of the ligands in the dataset were ranked higher than the decoys. As for the enrichment factor (EF) percentiles, we evaluated our data based on $\mathrm{EF}_{1 \%}$ or lower. The $\mathrm{EF}_{1 \%}$ of the conformer 1 for TanimotoCombo was found to be 28.1 (see Fig. S1†). However, matrices analysis for virtual screening based on EON_ET indicated relatively lower AUC of 0.649 with $\mathrm{EF}_{1 \%}$ of 21.4.

In the case of SBVS, the AUC was observed to reduce to 0.546 with $\mathrm{EF}_{1 \%}$ 6.7, possibly due to the larger active site of GPER-1, thus having the ability to interact with diverse molecular structures. However, SBVS statistical matrices AUC was improved from 0.546 to 0.660 along with the improvement in enrichment factor to 7.6 at the false positive rate of $1 \%$ upon docking of only selective GPER-1 ligand (G1, G15, and G36) and their decoys (Fig. 3B).

Overall, the retrospective validation results have supported our approach in the sequential screening of database molecules using LBVS followed by SBVS. The approach is robust to discriminate between active and decoy GPER-1 ligands.

\subsection{Ligand-based virtual screening (LBVS)}

3.2.1 Shape-based virtual screening. The shape-based screening (SBS) approach has been established as an essential virtual screening technique. The approach utilizes the concept of shape and electrostatic potential similarity to select new molecules which may show similar binding modes into the active site. The SBS is a useful tool for the identification and optimization of novel inhibitors with high potency and more selectivity. ${ }^{39,40}$ Besides, SBS does not require a target 3D structure or a well-developed SAR model that is necessary to create a reliable pharmacophore model, but only a known active compound is needed as input.

Shape-based virtual screening was carried out using ROCS. ROCS is a shape-based method for rapid similarity analysis of molecules. After a thorough literature assessment for GPER-1 ligands, G-1 (GPER-1 selective and potent agonist) was selected as the query compound for the generation of shapebased screening model. The pharmacophoric features of G-1 generated is depicted in Fig. 4. We hypothesized that screening hits with a similar molecular shape to G-1 would have an excellent GPER-1 modulation activity. ${ }^{41}$

In this analysis, ROCS color function assigned ten distinct pharmacophore features to G-1 query model, in which tetrahydro-3H-cyclopenta $[c]$ quinoline along with benzo[ $d][1,3]$ dioxole core was well-defined by five ring features. The query model presented with three hydrogen bond acceptor features from the two oxygen atoms in benzo[ $d][1,3]$ dioxole core, and one oxygen atom of the acetophenone group. Also, the amine and bromine (Br) served as a hydrogen-bond donor and hydrophobic feature respectively.

The generated ROCS query model was then applied in the virtual screening of large database of eMolecules library. The structural complementarity between the template and the screened molecules was evaluated by TanimotoCombo score (ROCS_TC) consisting of both ShapeTanimoto (ROCS_ST) and the ColorTanimoto (ROCS_CT). After shape-based virtual screening using ROCS_TC, top 5000 hits were retained for further in silico screening. Result analysis depicted that the highest ROCS_TC score of 1.861 for the top hit compound while the lowest ROCS_TC score was 1.204 as shown in Fig. 5.

During the screening, ROCS compares database compounds and the query by aligning the compounds and calculating the similarities including their volumes and chemical features. The similarity is evaluated and represented by a TanimotoCombo score, ranging from 0 to 2 . With TanimotoCombo score close to 2 , molecules will have an excellent shape and chemical-feature
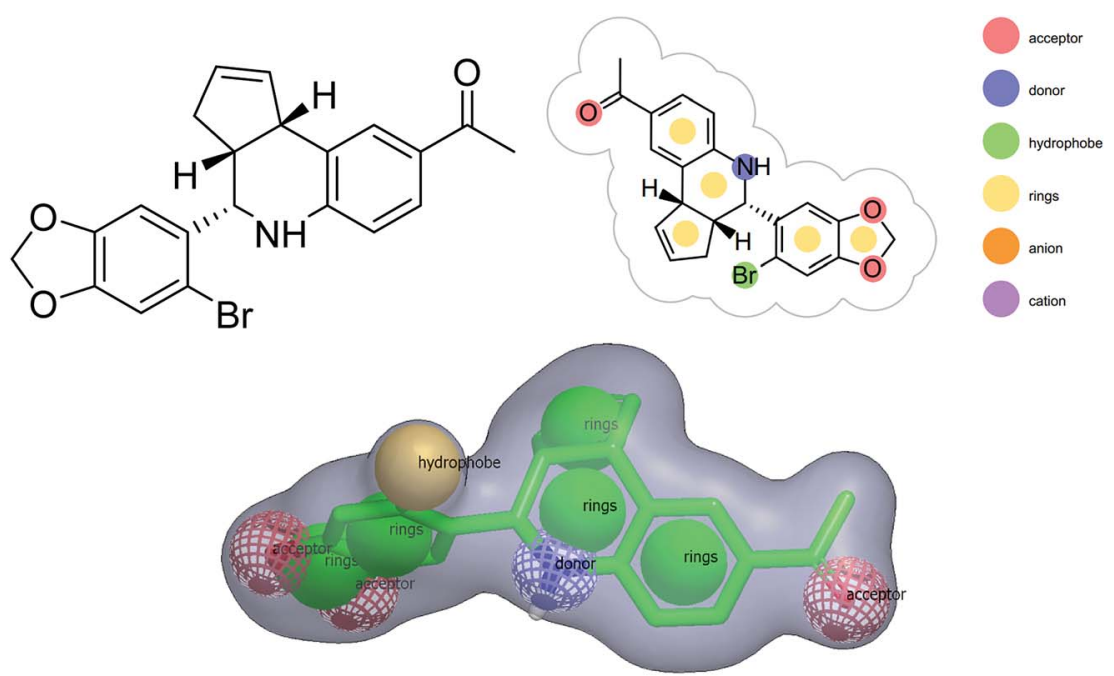

Fig. 4 Ligand-based pharmacophoric features of G-1. 


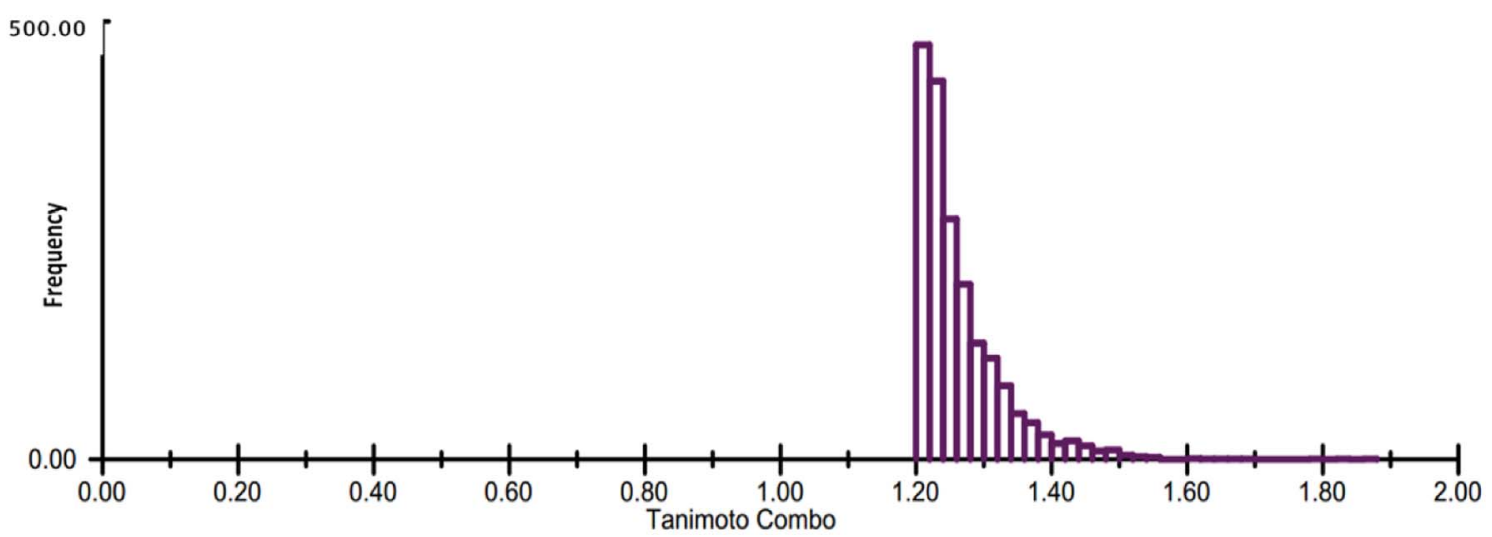

Fig. 5 Histogram representation of the ROCS_TanimotoCombo (ROCS_TC) score distribution for the top 5000 hits.

match, while the value close to 0 implies poor shape and chemical-feature dissimilarities.

3.2.2 Electrostatic potential similarity. In EON screening, a single lowest-energy conformer of G-1 was selected as a query model and used for all the electrostatic potential comparisons with that of ROCS hits compounds. The ranking of ligands was based on EON_Combo score which is a combination of ShapeTanimoto (ST) and PB Electrostatic Tanimoto (ET_pb). Analysis of EON hits of screen compounds revealed that the highest EON_Combo score of 1.651 (B) while the lowest EON potential score was 1.112 (C) (Fig. 6).

\subsection{Structure-based virtual screening (SBVS)}

\subsubsection{Homology modeling using Discovery Studio}

3.3.1.1 Template selection. Template selection and alignment are the preliminary steps in which the intended program or server relates the sequence of query with the template structure deposited in Protein Data Bank (PDB). The most widely used server in this process is Basic Local Alignment Search Tool (BLAST) which compared the sequence of query protein to the sequence in databases. The search identified thirty-six different templates and the CXCR4 chemokine receptor (PDB ID: 3ODU) with the resolution of $2.50 \AA$ was the
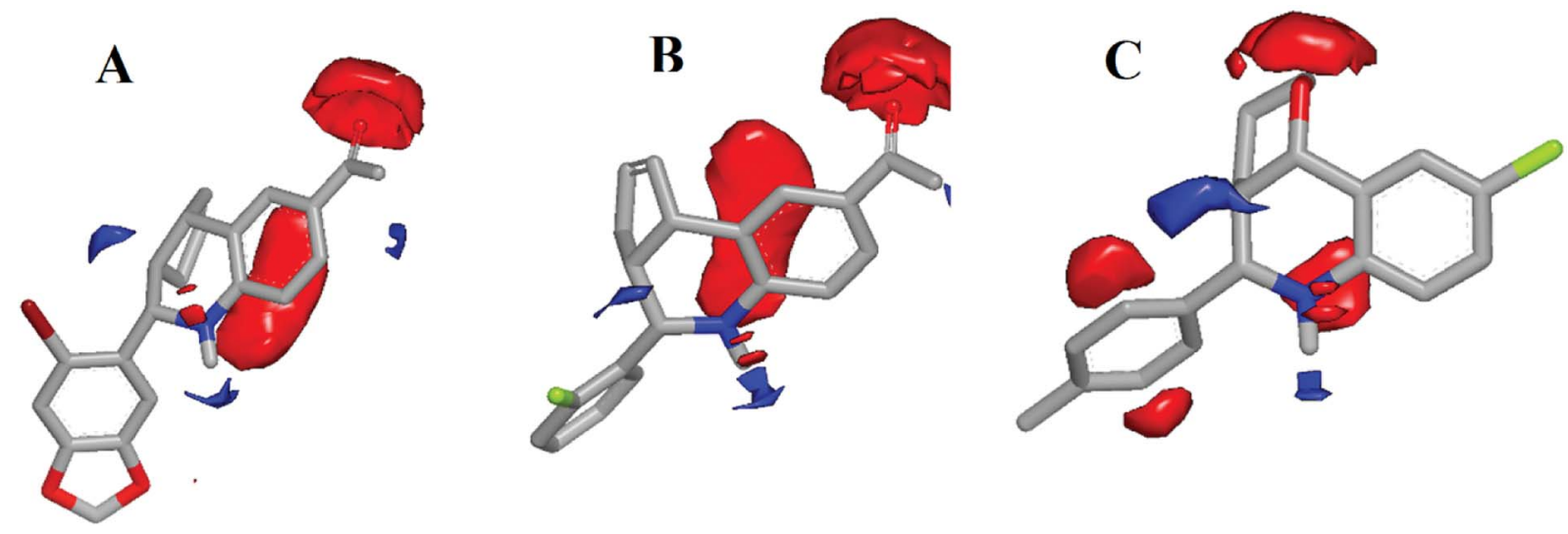

Fig. 6 EON Combo score of hits in comparison to G1. (A) G1 at extreme left has default EON_TC score of 2, (B) the top hits (score 1.651) and (C) the lowest hits (score 1.112). Red color indicates the electronegative area while blue colour indicated the electropositive area.

Table 1 Selected Template used for generation of Homology model

\begin{tabular}{|c|c|c|c|c|}
\hline Protein name & Template (PDB ID) & Sequence similarity & $\begin{array}{l}\text { Resolution } \\
\text { (̊) }\end{array}$ & Reference \\
\hline $\begin{array}{l}\text { CXCR4 chemokine } \\
\text { receptor }\end{array}$ & $3 \mathrm{ODU}$ & $46 \%$ & 2.50 & 42,43 \\
\hline Bovine rhodopsin & 1U19 & $40 \%$ & 2.2 & $44-49$ \\
\hline Bovine rhodopsin & $1 \mathrm{~F} 88$ & $24.6 \%$ & 2.8 & 8 \\
\hline$\beta 2$-Adrenergic GPCR & 2RH1 (in-active state) & $53 \%$ & 2.4 & 50 \\
\hline$\beta 2$-Adrenergic GPCR & 3SN6 (active state) & $76 \%$ & 3.2 & 50 \\
\hline
\end{tabular}


Table 2 DOPE score of Generated Homology Models using Modeller in Discovery Studio

\begin{tabular}{ll}
\hline Template PDB ID & DOPE score \\
\hline 3ODU & -33877.62 \\
1U19 & -27164.73 \\
1F88 & -40670.10 \\
Active state 3SN6 & -32334.34 \\
Inactive state 2RH1 & -36654.12
\end{tabular}

best template with $46 \%$ sequence similarity. The best hit template was compared with previously reported templates based on the sequence similarity, $E$-value, resolutions, cocrystallized ligands as shown in Table 1.

3.3.1.2 Alignment of query and template sequence. All templates listed in Table 1 were selected and aligned with GPER-1 sequence (Q99527) using the alignment tool in Discovery studio.

3.3.1.3 Homology models generation using Modeller. After the alignment of query and template sequence, Built-in Modeller tool in Discovery Studio was used for the generation of GPER-1 homology models. Top 20 models were generated for each template. These models were then analysed based on Discrete Optimized Protein Energy (DOPE) score and a model with the lowest DOPE score for each template was selected as the best model. Table 2 illustrates DOPE scores of all best models of each template.

3.3.1.4 Homology modeling of GPER-1 using online servers. Addition to Modeller tool in Discovery studio, models were generated using online servers such as SWISS-MODEL, ITASSER, and GPCR-MODSIM. GPER-1 models were obtained using default settings from both SWISS-MODEL and I-TASSER. Among online servers, only GPCR-MODSIM server allows the full optimization of sequence comparison for each topological region, that is, ( $\mathrm{N}$-terminal $(\mathrm{N}-\mathrm{t})$, transmembrane1 (TM1), intracellular1 (IL1), TM2, extracellular (EL1), TM3, IL2, TM4, EL2, TM5, IL3, TM6, EL3, TM7, C-t). GPCR-MODSIM identified 20 different templates based on sequence identity for each topological region as listed in Fig. 7. Based on the highest sequence identity percentage, five templates (4YAY, $4 \mathrm{DJH}$, 3OE0, 4DKL, 3ODU) were identified as the best templates and used for generating of the GPER-1 homology model.

3.3.1.5 Assessment and refinement of all generated models. Ramachandran plots of models were generated by RAMPAGE, to assess all generated models. The refinement of generated models was then carried out using ModRefiner server. Table 3 presents a comparative analysis of Ramachandran plots before and after refinement. Each model displayed improvement in the stereochemistry after refinement as revealed by the comparatively smaller number of residues in the outlier region. Due to a smaller number of amino acid residues in the outlier region, the number of residues becomes comparatively higher in both favoured and allowed region.

3.3.2 Selection of best homology model among all generated models. Based on a comparative analysis of Ramachandran plot obtained after refinement, a model generated using the multi-template approach on GPCR MODSIM server was shown to be of highest quality model. It presented $96.8 \%$ amino acid residues in the favored region and $2.9 \%$ residues in the allowed region with $0.3 \%$ of amino acid residues in the outlier region (Table 3). The 3D representation of the best model and its corresponding Ramachandran plot before and after refinement was shown in Fig. 8. This model was further used in the prediction of the binding pocket of GPER-1 and subsequent molecular docking studies.

3.3.3 Binding site identification of protein. After generation of the homology model, binding site identification is often an additional prerequisite requirement for performing SBVS. In

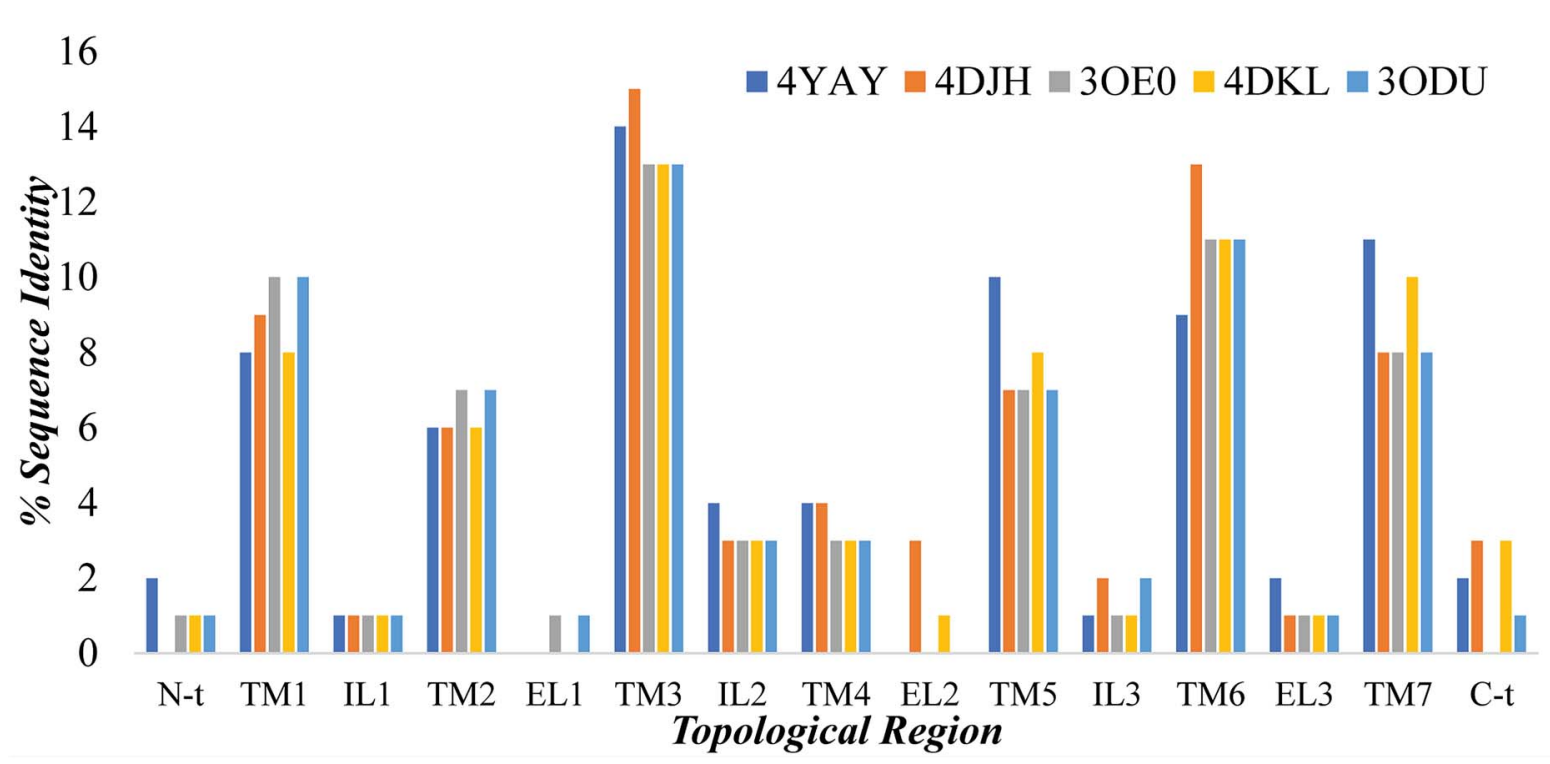

Fig. 7 The percentage of GPER-1 protein sequence identity with five selected templates (4YAY, 4DJH, 3OE0, 4DKL, 3ODU) at topological regions; $\mathrm{N}$-terminal ( $\mathrm{N}$-t), transmembrane1 (TM1), intracellular loop (IL), extracellular loop (EL) and C-terminal (C-t). 
Table 3 Comparative analysis of Ramachandran plot BEFORE and AFTER refinement of the homology model

\begin{tabular}{|c|c|c|c|c|c|c|}
\hline \multirow[b]{2}{*}{ Template PDB ID } & \multicolumn{3}{|l|}{ Before refinement } & \multicolumn{3}{|l|}{ After refinement } \\
\hline & $\begin{array}{l}\text { Number of } \\
\text { residues } \\
\text { in favoured region }\end{array}$ & $\begin{array}{l}\text { Number of } \\
\text { residues } \\
\text { in allowed region }\end{array}$ & $\begin{array}{l}\text { Number of } \\
\text { residues } \\
\text { in outlier region }\end{array}$ & $\begin{array}{l}\text { Number of } \\
\text { residues } \\
\text { in favoured region }\end{array}$ & $\begin{array}{l}\text { Number of } \\
\text { residues } \\
\text { in allowed region }\end{array}$ & $\begin{array}{l}\text { Number of } \\
\text { residues } \\
\text { in outlier region }\end{array}$ \\
\hline 3ODU (DS) & $323(95.3 \%)$ & $11(3.2 \%)$ & $5(1.5 \%)$ & 331 (97.6\%) & $6(1.8 \%)$ & $2(0.6 \%)$ \\
\hline 1U19 (DS) & $305(85.4 \%)$ & $34(9.5 \%)$ & $18(5.0 \%)$ & $325(91.0 \%)$ & $23(6.4 \%)$ & $9(2.5 \%)$ \\
\hline $1 \mathrm{~F} 88(\mathrm{DS})$ & $321(90.2 \%)$ & $22(6.2 \%)$ & $13(3.7 \%)$ & $339(95.2 \%)$ & $14(3.9 \%)$ & $3(0.8 \%)$ \\
\hline Active state 3SN6 (DS) & $354(94.9 \%)$ & $13(3.5 \%)$ & $6(1.6 \%)$ & $364(97.6 \%)$ & $7(1.9 \%)$ & $2(0.5 \%)$ \\
\hline Inactive state 2RH1 (DS) & $360(96.5 \%)$ & $9(2.4 \%)$ & $4(1.1 \%)$ & $368(98.7 \%)$ & $4(1.1 \%)$ & $1(0.3 \%)$ \\
\hline 3ODU (SWISS-MODEL) & $258(92.8 \%)$ & $18(6.5 \%)$ & $2(0.7 \%)$ & $269(96.8 \%)$ & $7(2.5 \%)$ & $2(0.7 \%)$ \\
\hline I-TASSER & $292(78.3 \%)$ & $51(13.7 \%)$ & $30(8.0 \%)$ & $352(94.4 \%)$ & $14(3.8 \%)$ & $7(1.9 \%)$ \\
\hline 3ODU (GPCR MODSIM) & $348(93.3 \%)$ & $21(5.6 \%)$ & $4(1.1 \%)$ & $360(96.5 \%)$ & $10(2.7 \%)$ & $3(0.8 \%)$ \\
\hline $\begin{array}{l}\text { Multi template (GPCR } \\
\text { MODSIM) }\end{array}$ & $355(95.2 \%)$ & $14(3.8 \%)$ & $4(1.1 \%)$ & $361(96.8 \%)$ & $11(2.9 \%)$ & $1(0.3 \%)$ \\
\hline
\end{tabular}
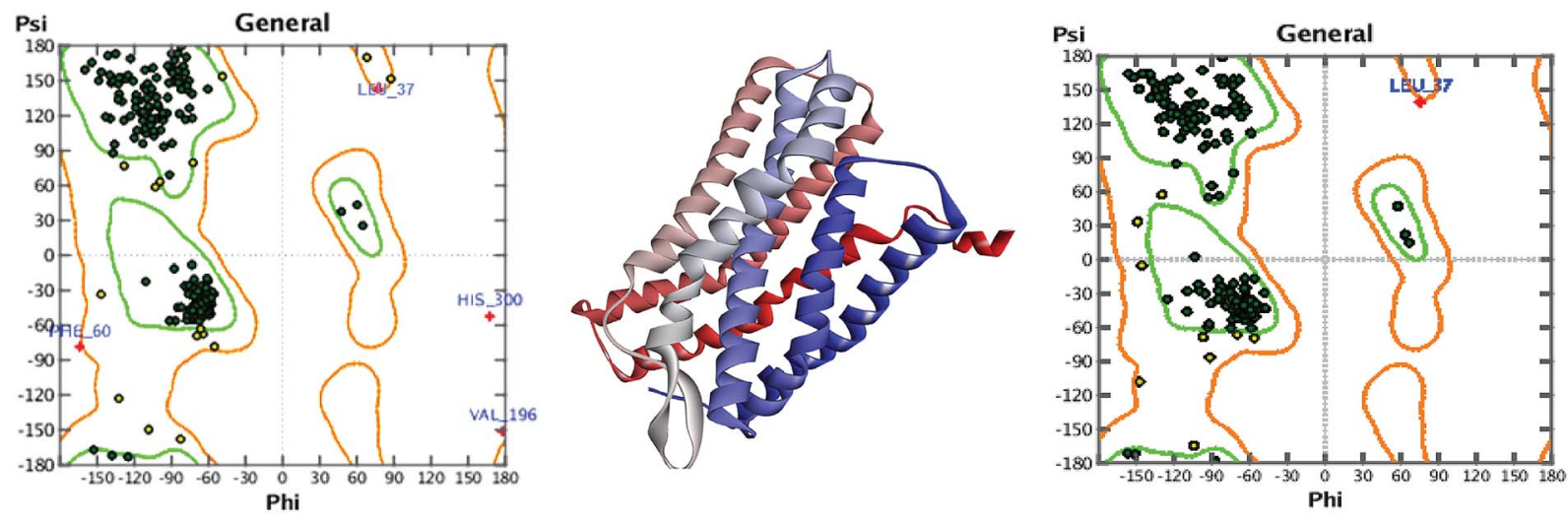

Fig. 8 The 3D structure representation of the best GPER-1 model (in the middle). Ramachandran plot representation of GPER-1 model before and after refinement are shown in the left and right side respectively.

this study, CASTp (Computed Atlas of Surface Topography of proteins) server was used and predicted a total of 40 catalytic sites in GPER-1 receptor. The best catalytic site was noted with the solvent accessible (SA) area of $1477.1 \AA$ and the volume of $1396.0 \AA$, while the rest of binding sites was shown with the surface area of less than $140 \AA$. Therefore, the predicted binding pocket comprising 73 amino acid residues was considered as the best catalytic site. In addition, the topological regions of these selected amino acid residues were identified for further docking studies. The amino acid residues within active pocket GPER-1 were tabulated in Table 4.

3.3.4 Docking-based virtual screening. To further narrow down the hits obtained from ROCS and EON screening, molecular docking of all compounds was performed within the active site using FRED docking software. The FRED program has a good application record in structure-based drug discovery. Recently, Huabin Hu et al. identified FRED (Chemgauss4 score) as the best docking score among three different docking score functions. ${ }^{51} \mathrm{~A}$ maximum of 200 conformers per ligand was generated and used as a set of input. Docking of reported GPER1 ligands and selected virtual hit compounds were performed using the same protocol as described in method 2.3.3. A maximum of 10 poses for each compound were generated, and the pose with the lowest Chemgauss4 score was selected.

The Chemgauss 4 score of the best binding pose of GPER-1 selective ligands (G1, G-36, and G-15) was $-11.5754,-11.3850$ and -9.1130 respectively. Docking studies of virtual hits obtained from ROCS and EON indicated that the docking score of the top 100 ROCS and EON hit compounds were higher than that of selective ligands of GPER-1. Among the top 100 compounds, 1-((3aS,4S,9bR)-4-(3-fluorophenyl)-3a,4,5,9b-tetrahydro-3H-cyclopenta[c]quinolin-8-yl)ethan-1-one (code: 6120497_SRS_10) exhibited the highest score of -14.67. Upon comparison, the best scoring poses of docked ligands were noted to have the same docking orientations as GPER-1 selective ligands.

3.3.5 Comparative binding interaction analysis of docked poses. Molecular docking was carried out to gain insight into the binding interactions of reported GPER-1 ligands within the active pocket of the target. Detailed binding interactions of GPER-1 ligands are shown in Fig. 9. Analysis of best-docked pose of G-1 within the active site of GPER-1 revealed the presence of a conventional hydrogen bond between the amino acid residue Asn276 and carbonyl oxygen of acetophenone moiety of 
Table 4 Solvent Accessible (SA) area, volume and interacting amino acid residue in the active site predicted by CASTp

\begin{tabular}{|c|c|c|c|}
\hline Active site & Area (SA) & Volume (SA) & $\begin{array}{l}\text { Interacting amino acid residues } \\
\text { within the active pocket of GPER-1 }\end{array}$ \\
\hline 1 & 1477.1 & 1396.0 & $\begin{array}{l}\text { Leu49, Ser50, His52, Gln53, Gln54, } \\
\text { Ile57, Gly58, Leu61, Ser62, Tyr65, } \\
\text { Phe68, Leu108, Val109, Asp111, } \\
\text { Ser112, Ile114, Glu115, Val116, } \\
\text { Asn118, Leu119, His120, Arg122, } \\
\text { Tyr123, Cys130, Met133, Ser134, } \\
\text { Leu137, Gln138, Asn140, Met141, } \\
\text { Ser144, Val145, Pro192, Val196, } \\
\text { His200, Asp202, Ala204, Cys205, } \\
\text { Phe206, Cys207, Phe208, Asp210, } \\
\text { Arg212, Glu213, Val214, Gln215, } \\
\text { Trp216, Glu218, Val219, Gly222, } \\
\text { Phe223, Ile224, Phe268, Phe269, } \\
\text { Trp272, Leu273, Glu275, Asn276, } \\
\text { Phe278, Ile279, Ser280, His282, } \\
\text { Leu283, Arg286, Phe298, His300, } \\
\text { Ala301, His302, Leu304, Thr305, } \\
\text { Asn310, Leu311, Phe314, Asn316, } \\
\text { Ser317 }\end{array}$ \\
\hline
\end{tabular}

G1. This type of hydrogen bonding is also previously reported. ${ }^{42,52}$

Besides, hydrogen bonding network was formed by amino acid Glu218 with NH group of G1. Moreover, the amino acid residue of Asp210 contributed in hydrogen bond network to one of the oxygens in dioxole moiety, while Glu218 and Gln138 were involved in forming pi-anion and pi-donor hydrogen bond respectively with the aromatic ring of benzodioxole moiety of G1. Additional hydrophobic interactions were formed by the amino acid residues of Ile279, Met141, Leu137. In this hydrophobic interaction Leu137, Met141 were making hydrophobic interaction with the bromine atom of $\mathrm{G}-1$.
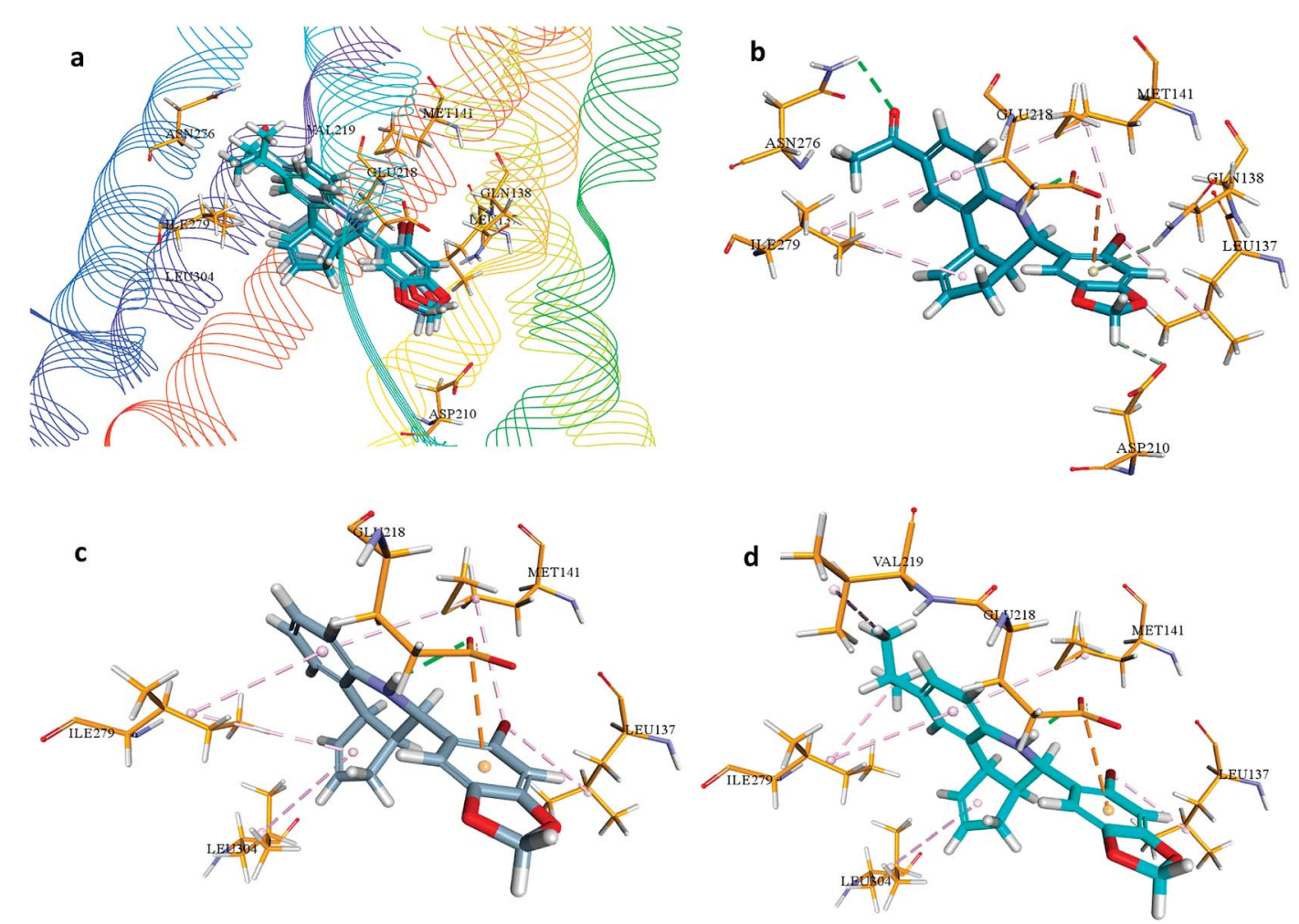

Fig. 9 Binding interactions of GPER-1 ligands. (a) Superimposition of best-docked poses of G1, G-15, and G-36 displayed in blue, grey, and cyan respectively. (b) Binding interactions of G1, (c) binding orientation of G15 and (d) binding interaction of G36 with the amino acids of GPER-1 (golden). Hydrogen bonding, electrostatic and hydrophobic bonds are shown in green, yellow and pink color dotted lines respectively. Amino acid residues are shown in golden sticks. 

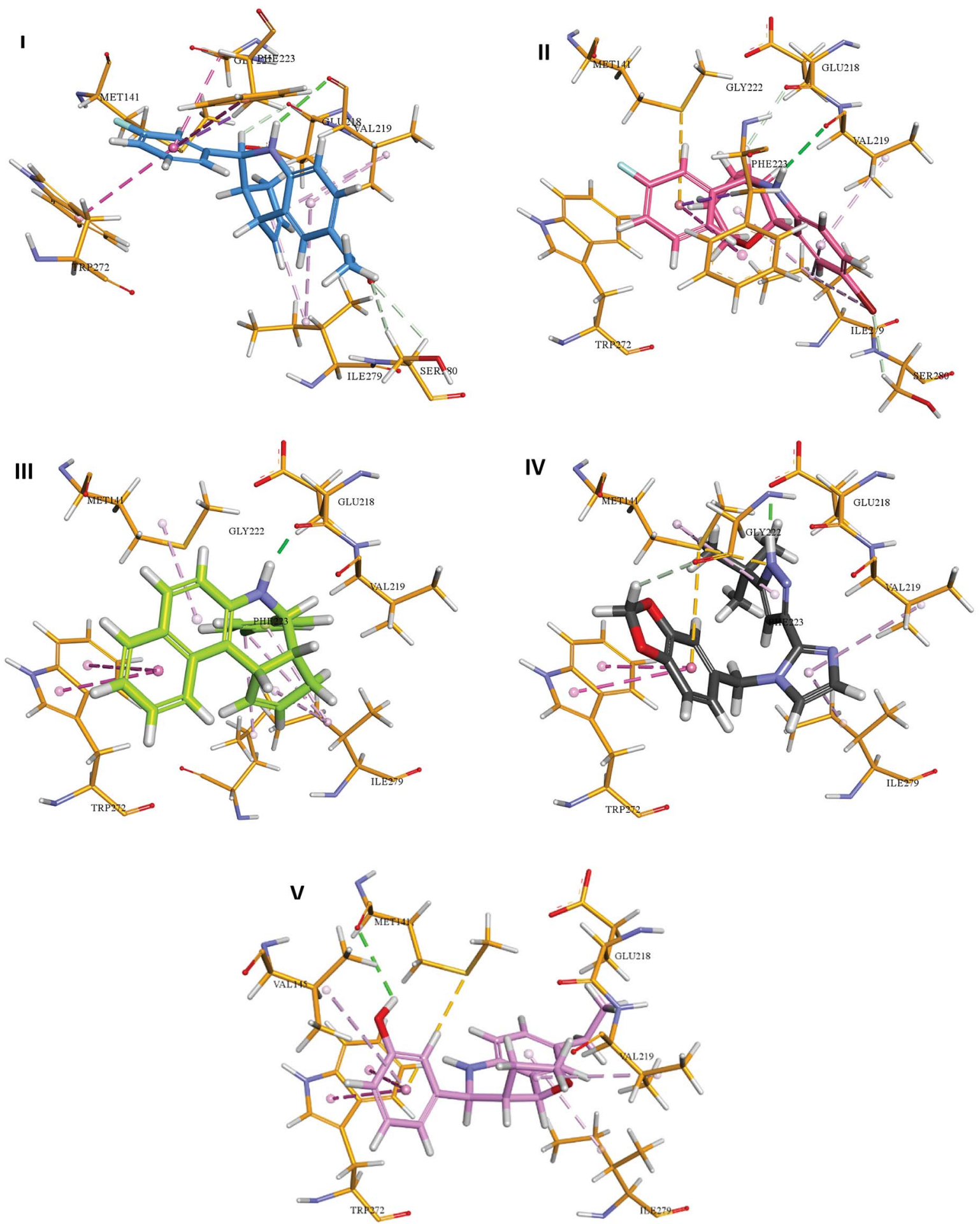

Fig. 10 Binding interactions of top hits compound in each identified scaffold within the active site of GPER-1. Hydrogen bond interactions are shown in green dotted line while hydrophobic interactions are shown in pink dotted line.

Binding analysis of G-36 depicted hydrogen bond interaction between Gln218 and NH moiety of G36, pi-anion interaction between $\operatorname{Gln} 218$ and the aromatic ring of benzoxazole moiety, and hydrophobic interaction between Leu137 and the bromine atom of G36. Moreover, other electrostatic and hydrophobic interactions were formed by amino acid residues Leu304, Ile279, Val219, Leu137, Met141, and Ile279.
In the binding analysis of G-15, the orientation of G-15 was noted to be similar to that of G1 and G36. Amino acid residues involved in hydrogen bonding and hydrophobic interaction of G-15 were alike G-36, but due to the absence of isopropyl moiety in G-15, the hydrophobic interaction with this moiety was not observed in G-15. 
Table 5 Compound code in the database, chemical structures, FRED Chemgauss 4 score, electrostatic potential combo score and ROCS TanimotoCombo scores of top five best-identified scaffolds

The analysis of docking calculation resulted in about 100 top hits which had higher Chemgauss4 score compared to the GPER-1 selected ligands: G1 (query compound), G15 and G36. Based on the Chemgauss4 score, five different scaffolds (shown in Table 5) were identified in top 100 hits, namely, $(3 a S, 4 S, 9 b R)$-4-phenyl-3a,4,5,9b-tetrahydro-3 $H$-cyclopenta[c] quinoline (compound 6120497_SRS_10), (4aS,5S,10bS)-5phenyl-3,4,4a,5,6,10b-hexahydro-2 $H$-pyrano[3,2-c]quinoline (compound 27191896_S_0), (3aR,4S,11cS)-4-phenyl$3 a, 4,5,11 c$-tetrahydro-3H-benzo[f]cyclopenta[c]quinoline (compound 1275536_RSS), 3-(1-(benzo[d] [1,3]dioxol-5ylmethyl)-1 $H$-imidazol-2-yl)-1 $H$-pyrazole (compound 36597047_125) and (3aS,4S,9bS)-4-phenyl-2,3,3a,4,5,9b-hexahydrofuro[3,2-c]quinoline (compound 32861528_S_2) Fig. 10 shows the binding interactions of top hits compounds listed in Table 5.

Interestingly, the topmost identified scaffolds in the present study, namely $(3 a S, 4 S, 9 b R)-4$-phenyl-3a,4,5,9b-tetrahydro- $3 H$ cyclopenta[c]quinoline are reported in US patent in 2008 by $\mathrm{E}$. R. Prossnitz et al. A comparison of the amino acid residues involved in the binding of the best hits and GPER-1 selective ligands revealed that seven different amino acid residues (Met141, Val219, Gly222, Phe223, Trp272, Ile279 and Ser280) were forming hydrophobic interaction while amino acid residue Glu218 was involved in forming hydrogen bond with amine group of hit compounds.
In agreement with our results, recently in 2018 Cao L.-Y. et al. has shown involvement of almost the same amino acid residues in the binding of GPER-1 ligands at the active site of GPER-1.,53 Similar to their observation, in the present study binding of the best scaffold compound, $(3 a S, 4 S, 9 b R)$-4-phenyl-3a,4,5,9b-tetrahydro-3H-cyclopenta[c]quinoline, $\quad(4 a S, 5 S, 10 b S)$-5-phenyl3,4,4a,5,6,10b-hexahydro-2 $H$-pyrano[3,2-c]quinoline, $(3 a R, 4 S, 11 c S)$-4-phenyl-3a,4,5,11c-tetrahydro-3H-benzo[f]cyclopenta $[c]$ quinoline, 3-(1-(benzo[ $d][1,3]$ dioxol-5-ylmethyl)- $1 H$-imidazol-2-yl)- $1 H$-pyrazole and ( $3 a S, 4 S, 9 b S)$-4-phenyl-2,3,3a,4,5,9bhexahydrofuro[3,2-c] quinoline at the active site of the GPER-1 formed similar hydrophobic interactions thereby signifying the validation of our in silico virtual screening approach.

\subsection{Prospective validation of virtual screening}

3.4.1 Synthesis of hit compounds. Fig. 11 illustrates the general scheme for the synthesis of SKo and SKOP. The compounds were synthesized using microwave irradiation with slight modification from previously report. ${ }^{36}$ The reaction condition was optimized at $100{ }^{\circ} \mathrm{C}$ for $20 \mathrm{~min}$. The target compounds were obtained in better yield with higher stereoselectivity compared to the conventional synthesis.

3.4.2 Antiproliferative activities of hit compounds using GPER-1 specific cell lines. Antiproliferative activities of compound G-1, SK0, and SKOP were evaluated using GPER-1 specific cell lines and the $\mathrm{EC}_{50}$ are tabulated in Table 6 . 


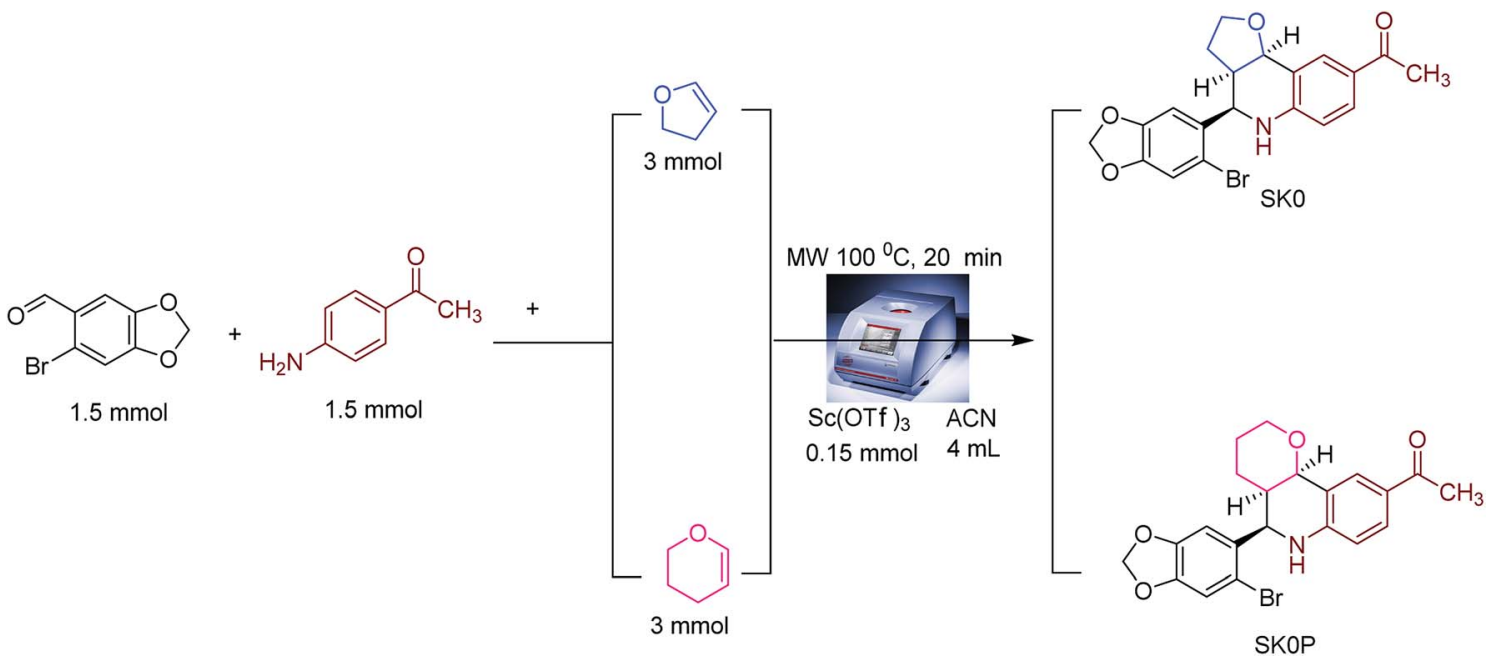

Fig. 11 General scheme for the synthesis of SKO and SKOP using microwave irradiation.

Table 6 Antiproliferative activities of compound G-1, SKO and SKOP against GPER-1 specific cell lines and molecular docking score of the investigated compounds with GPER-1

\begin{tabular}{|c|c|c|c|}
\hline \multirow[b]{2}{*}{ Compound codes } & \multicolumn{2}{|c|}{$\underline{\mathrm{EC}_{50}(\mu \mathrm{M})}$} & \multirow{2}{*}{$\begin{array}{l}\text { Chemguass } 4 \\
\text { scores }\end{array}$} \\
\hline & SK-BR-3 & MCF-7 & \\
\hline G-1 & 0.54 & 39.92 & -11.580 \\
\hline SKO & 2.77 & 6.69 & -11.690 \\
\hline SKOP & 9.26 & 10.83 & -11.000 \\
\hline
\end{tabular}

Selection of SK-BR-3 and MCF-7 cell lines was based on the previously reported literature, in which these cell lines were specifically used to evaluate the antiproliferative activities of GPER-1 agonists. ${ }^{37,38}$ It is worthy to note that all three compounds presented similar FRED Chemguass4 score as the top hits obtained from the aforementioned virtual screening studies.

Among the compounds, G1 exhibited anti-proliferative activities against SK-BR-3 and MCF7 having EC $_{50}$ value of 0.54 $\mu \mathrm{M}$ and $39.92 \mu \mathrm{M}$ respectively. Relatively, SKO and SKOP elicited higher antiproliferative activities in SK-BR-3 cell lines $\left(\mathrm{EC}_{50}=\right.$ 2.77) than in MCF-7 cell lines. Likewise, SKOP presented EC $_{50}$ value of $9.26 \mu \mathrm{M}$ in SK-BR-3 cell lines which were higher than in MCF 7. The observation of better antiproliferative activity in SKBR-3 breast cancer cell lines is in positive relationship with its higher expression of GPER-1 protein compared to MCF-7 cell lines. Therefore, hits identified from eMolecules using our adopted sequential ligand and structure-based virtual screening can be considered worthy for further screening of biological activity against GPER-1 protein.

\section{Conclusion}

In the present study, we demonstrated that the adopted in silico virtual screening protocol is an indispensable tool for hit identification in the domain of small molecule GPER-1 modulators. We reported herein the sequential ligand-based and structure-based virtual screening procedure that can be employed in the identification of new GPER-1 modulators. Retrospective metrics and the in vitro evaluation of antiproliferative activity validated the model. Starting from the retrospective validation of virtual screening procedure, followed by the virtual screening of commercial database of 7 million compounds, only 100 compounds were identified as potential GPER-1 ligands. Interestingly, the identified molecules possessing similar scaffolds which were reported as potent GPER-1 ligands in literature. The antiproliferative activity of two compounds representing the top-ranked scaffold further confirmed that the virtual screening protocol developed in this study is reliable and worthwhile.

\section{Conflicts of interest}

The authors declare that they have no competing financial interests.

\section{Acknowledgements}

We are grateful to the anonymous referees for their informative comments, suggestions and insights for improving this manuscript. The authors would like to thank Ministry of Higher Education (FRGS, grants no. FRGS/1/2015/ST01/MUSM/03/1) for financial support. We also thanks OpenEye Scientific Software for providing a free academic license to perform in silico studies.

\section{References}

1 M. O'Hayre, M. S. Degese and J. S. Gutkind, Curr. Opin. Cell Biol., 2014, 27, 126-135.

2 A. S. Hauser, M. M. Attwood, M. Rask-Andersen, H. B. Schiöth and D. E. Gloriam, Nat. Rev. Drug Discovery, 2017, 16, 829.

3 E. R. Prossnitz and M. Barton, Nat. Rev. Endocrinol., 2011, 7, 715. 
4 M. Maggiolini and D. Picard, J. Endocrinol., 2010, 204, 105114.

5 L.-Y. Cao, X.-M. Ren, Y. Yang, B. Wan, L.-H. Guo, D. Chen and Y. Fan, Environ. Health Perspect., 2018, 126, 069001.

6 S. Vilar, G. Cozza and S. Moro, Curr. Top. Med. Chem., 2008, 8, 1555-1572.

7 L. Tan, H. Geppert, M. T. Sisay, M. Gütschow and J. Bajorath, ChemMedChem, 2008, 3, 1566-1571.

8 C. Rosano, R. Lappano, M. F Santolla, M. Ponassi, A. Donadini and M. Maggiolini, Curr. Med. Chem., 2012, 19, 6199-6206.

9 S. Sandhaus, P. P. Chapagain and Y.-C. Tse-Dinh, Sci. Rep., 2018, 8, 1437.

10 E. D. Brown and G. D. Wright, Nature, 2016, 529, 336.

11 R. S. Ferreira, R. V. Guido, A. D. Andricopulo and G. Oliva, Expert Opin. Drug Discovery, 2011, 6, 481-489.

12 H. Eckert and J. Bajorath, Drug discovery today, 2007, 12, 225233.

13 A. D. Andricopulo, R. V. Guido and G. Oliva, Curr. Med. Chem., 2008, 15, 37-46.

14 P. Kolb, D. M. Rosenbaum, J. J. Irwin, J. J. Fung, B. K. Kobilka and B. K. Shoichet, Proc. Natl. Acad. Sci. U. S. A., 2009, 106, 6843-6848.

15 C. N. Cavasotto, W. Orry and J. Andrew, Curr. Top. Med. Chem., 2007, 7, 1006-1014.

16 D. R. Weiss, A. Bortolato, B. Tehan and J. S. Mason, J. Chem. Inf. Model., 2016, 56, 642-651.

17 A. Cereto-Massagué, L. Guasch, C. Valls, M. Mulero, G. Pujadas and S. Garcia-Vallvé, Bioinformatics, 2012, 28, 1661-1662.

18 T. Sterling and J. J. Irwin, J. Chem. Inf. Model., 2015, 55, 23242337.

19 M. M. Mysinger, M. Carchia, J. J. Irwin and B. K. Shoichet, J. Med. Chem., 2012, 55, 6582-6594.

20 A. N. Jain and A. Nicholls, J. Comput.-Aided Mol. Des., 2008, 22, 133-139.

21 A. K. Akobeng, Acta Paediatr., 2007, 96, 644-647.

22 S. Lätti, S. Niinivehmas and O. T. Pentikäinen, J. Cheminf., 2016, 8, 45.

23 P. C. Hawkins, A. G. Skillman and A. Nicholls, J. Med. Chem., 2007, 50, 74-82.

24 P. C. Hawkins, A. G. Skillman, G. L. Warren, B. A. Ellingson and M. T. Stahl, J. Chem. Inf. Model., 2010, 50, 572-584.

25 P. Thomas, Y. Pang, E. Filardo and J. Dong, Endocrinology, 2005, 146, 624-632.

26 EON. 2.2.0.5, OpenEye Scientific Software, Santa Fe, NM. http://www.eyesopen.com.

27 M. y. Shen and A. Sali, Protein Sci., 2006, 15, 2507-2524.

28 A. Roy, A. Kucukural and Y. Zhang, Nat. Protoc., 2010, 5, 725.

29 T. Schwede, J. Kopp, N. Guex and M. C. Peitsch, Nucleic Acids Res., 2003, 31, 3381-3385.

30 M. Esguerra, A. Siretskiy, X. Bello, J. Sallander and H. Gutiérrez-de-Terán, Nucleic Acids Res., 2016, 44, W455W462.

31 D. Xu and Y. Zhang, Biophys. J., 2011, 101, 2525-2534.

32 J. Dundas, Z. Ouyang, J. Tseng, A. Binkowski, Y. Turpaz and J. Liang, Nucleic Acids Res., 2006, 34, W116-W118.
33 M. McGann, J. Chem. Inf. Model., 2011, 51, 578-596.

34 OEDocking. 3.2.2, OpenEye Scientific Software, Santa Fe, NM, 2017, http://www.eyesopen.com.

35 E. F. Pettersen, T. D. Goddard, C. C. Huang, G. S. Couch, D. M. Greenblatt, E. C. Meng and T. E. Ferrin, J. Comput. Chem., 2004, 25, 1605-1612.

36 R. Burai, C. Ramesh, M. Shorty, R. Curpan, C. Bologa, L. A. Sklar, T. Oprea, E. R. Prossnitz and J. B. Arterburn, Org. Biomol. Chem., 2010, 8, 2252-2259.

37 C. Wang, X. Lv, C. He, G. Hua, M. Tsai and J. S. Davis, Cell Death Dis., 2013, 4, e869.

38 W. Wei, Z. Chen, K. Zhang, X. Yang, Y. Wu, X. Chen, H. Huang, H. Liu, S. Cai and J. Du, Cell Death Dis., 2014, 5, e1428.

39 D.-S. Kim, C.-M. Kim, C.-I. Won, J.-K. Kim, J. Ryu, Y. Cho, C. Lee and J. Bhak, J. Biomol. Struct. Dyn., 2011, 29, 219-242.

40 H.-P. Sun, J.-M. Jia, F. Jiang, X.-L. Xu, F. Liu, X.-K. Guo, B. Cherfaoui, H.-Z. Huang, Y. Pan and Q.-D. You, Eur. J. Med. Chem., 2014, 79, 399-412.

41 C. G. Bologa, C. M. Revankar, S. M. Young, B. S. Edwards, J. B. Arterburn, A. S. Kiselyov, M. A. Parker, S. E. Tkachenko, N. P. Savchuck and L. A. Sklar, Nat. Chem. Biol., 2006, 2, 207-212.

42 A. Bruno, F. Aiello, G. Costantino and M. Radi, Mol. Inf., 2016, 35, 333-339.

43 F. Aiello, G. Carullo, F. Giordano, E. Spina, A. Nigro, A. Garofalo, S. Tassini, G. Costantino, P. Vincetti and A. Bruno, ChemMedChem, 2017, 12, 1279-1285.

44 R. Lappano, C. Rosano, P. De Marco, E. M. De Francesco, V. Pezzi and M. Maggiolini, Mol. Cell. Endocrinol., 2010, 320, 162-170.

45 M. Pupo, A. Pisano, R. Lappano, M. F. Santolla, E. M. De Francesco, S. Abonante, C. Rosano and M. Maggiolini, Environ. Health Perspect., 2012, 120, 1177.

46 D. Méndez-Luna, M. Martínez-Archundia, R. C. Maroun, G. Ceballos-Reyes, M. Fragoso-Vázquez, D. González-Juárez and J. Correa-Basurto, J. Biomol. Struct. Dyn., 2015, 33, 2161-2172.

47 R. Lappano, C. Rosano, A. Pisano, M. F. Santolla, E. M. De Francesco, P. De Marco, V. Dolce, M. Ponassi, L. Felli and G. Cafeo, Dis. Models Mech., 2015, 8, 1237-1246.

48 R. Lappano, M. F. Santolla, M. Pupo, M. S. Sinicropi, A. Caruso, C. Rosano and M. Maggiolini, Breast Cancer Res., 2012, 14, R12.

49 V. Sarmiento, I. Ramirez-Sanchez, A. Moreno-Ulloa, D. Romero-Perez, D. Chávez, M. Ortiz, N. Najera, J. CorreaBasurto, F. Villarreal and G. Ceballos, Bioorg. Med. Chem. Lett., 2018, 28, 658-663.

50 C. K. Arnatt and Y. Zhang, Mol. Inf., 2013, 32, 647-658.

51 H. Hu, J. Xia, D. Wang, X. S. Wang and S. Wu, Int. J. Mol. Sci., 2017, 18, 137.

52 F. Aiello, G. Carullo, F. Giordano, E. Spina, A. Nigro, A. Garofalo, S. Tassini, G. Costantino, P. Vincetti and A. Bruno, ChemMedChem, 2017, 12, 1279-1285.

53 L.-Y. Cao, X.-M. Ren, C.-H. Li, J. Zhang, W.-P. Qin, Y. Yang, B. Wan and L.-H. Guo, Environ. Sci. Technol., 2017, 51, 11423-11430. 